\title{
Role of Dedicated Cardiac Emergency Unit in Early Identification and Management of Acute Myocardial Infarction in a Developing Country of South Asia
}

Farhala Baloch ${ }^{1}$, Amina Khan ${ }^{2}$, Ashmal Kabani ${ }^{3}$, Saulat Fatimi ${ }^{4}$, Javed Tai ${ }^{5}$, Aamir H. Khan ${ }^{1}$, Shiraz Hashmi $^{4}$, Mazeera Aslam 6

1. Medicine/Cardiology, The Aga Khan University, Karachi, PAK 2. School of Nursing, The Aga khan University, Karachi, PAK 3. Medicine, The Aga Khan University, Karachi, PAK 4. Cardiothoracic Surgery, The Aga Khan University, Karachi, PAK 5. Cardiology, The Aga Khan Hospital, Karachi, PAK 6. School of Nursing, The Aga Khan University, Karachi, PAK

Corresponding author: Farhala Baloch, farhala.baloch@googlemail.com

\section{Abstract}

\section{Background}

The care of patients presenting with chest pain to multidisciplinary services hospital gets compromised due to the busy triage system. A separate and specialized equipped cardiac emergency unit (CAR-ERU) can improve patient's outcomes.

\section{Objectives}

To enhance early recognition and treatment of acute myocardial infarction (AMI) patients. To sustain key performance quality indicators (KPIs) for AMI.

\section{Methods}

In October 2016, a separate CAR-ERU was established inside the multidisciplinary emergency department (MED). A dedicated specialized heart-lung and vascular teams were hired under the supervision of service line leadership. The KPIs that were identified benchmark with international practice guidelines. Data were collected and stored for analysis. Exemption from the ethical review committee was obtained.

Review began 10/13/2020 Review ended 10/27/2020 Published 11/10/2020

\section{() Copyright 2020}

Baloch et al. This is an open access article distributed under the terms of the Creative Commons Attribution License CC-BY 4.0., which permits unrestricted use, distribution, and reproduction in any medium, provided the original author and source are credited.

\section{Results}

A total of 2914 patients visited CAR-ERU from October 2016 to September 2017 for a period of one year. Out of which $30 \%$ were diagnosed with acute coronary syndrome (ACS) and this included $8 \%$ diagnosis with STsegment elevation myocardial infarction (STEMI). Over $98.8 \%$ of the electrocardiogram (ECG) was done within 10 minutes of arrival while aspirin was given to $96.5 \%$ of patients within one hour. The door to balloon time (DBT) of $<90$ min was achieved in $70 \%$ of patients. A significant reduction in length of stay in the emergency department and financial burden was noted. Sustainability of major KPI was observed over the subsequent years.

\section{Conclusion}

The introduction of a dedicated CAR-EU improved clinical outcomes, reduced length of stay and financial burden in AMI patients managed in CAR-EU. Our tertiary care hospital is the first one of its kind to take this quality initiative in a lower-middle-income country (LMIC) Pakistan.

Categories: Cardiology, Emergency Medicine, Quality Improvement

Keywords: acute myocardial infarction, cardiac emergency unit, key performance indicators, acute st-elevation myocardial infarction

\section{Introduction}

Chest pain accounts for about 5.6 million emergency department (ED) visits annually [1], Among these, early recognition of ST-segment elevation myocardial infarction (STEMI) cases is critical especially in a multidisciplinary hospital where a common and busy triage system receiving a myriad of patients with different complaints [2,3]. In developing countries, less focus has been given to emergency medical care [4]. The healthcare system in our country is at a progressive stage. Participation in various programs like making health policies, public-private partnerships and many others do exist but with a limited scope and there is a need to take strong initiatives [5].

Multiple evidences suggest that deaths are prevented and disabilities averted for diseases such as acute 
myocardial infarction (AMI), stroke, pregnancy and sepsis by strengthening emergency services, through the diagnostic protocols, decision aids, novel approach and other system changes [6,7]. AMI is one of the leading causes of death and accounts for $10.6 \%$ of hospital mortality and disability worldwide $[8,9]$. On the other hand mortality in STEMI is clearly related to the time of symptom onset to reperfusion time. Every patient who presents with symptoms suggestive of STEMI must have an electrocardiogram (ECG) done within 10 minutes of first medical contact (FMC) followed by a sequence of intervention including compliance to time-bound aspirin and reperfusion algorithms [10]. Our hospital has a precise vision of providing quality patient care. Although our multidisciplinary emergency department demonstrates encouraging results through organized approaches in terms of provision of prompt quality services, however, patients presenting with chest pain needs a quick response out of a busy triage counter dealing with hundreds of patients and variable symptoms on presentation.

The innovation followed by the implementation of the heart-lung and vascular service line in our institute brought forward a notion to establish a separate dedicated cardiac emergency unit (CAR-ERU) within the existing emergency department which would aim to early identification and treatment of STEMI patients and enhance the quality of care. This quality initiative step of providing a separate CAR-ERU in a multidisciplinary tertiary care hospital to improve patient outcomes is taken the first time in Pakistan.

\section{Materials And Methods}

A dedicated cardiac team of cardiac physicians and nurses were allocated for separate CAR-ERU. All patients age 18 and above of either gender presenting with signs and symptoms suggestive of acute myocardial infarction were immediately moved to this unit. The key performance quality indicators (KPIs) were identified that set a benchmark in accordance with international practice guidelines. The data for the KPIs were collected and analyzed by dedicated quality improvement committee (QIC) nurses and research officer. Monthly QIC meetings were conducted under the leadership for feedback and future directions.

The KPIs includes ECG within 10 minutes of FMC which is the emergency department in our scenario, aspirin within 60 minutes of arrival, the proportion of STEMI patients receiving revascularization within 90 minutes, the outcome matrices includes mortality and morbidity, length of emergency stay and costeffectiveness which ultimately leads to patient's satisfaction. The initial data for the period of one year from 2016 to 2017 were analyzed on a quarterly basis. The subsequent data to measure the sustainability of major KPIs are analyzed over the year 2018 and compared with 2019 data.

For continuous variables, we report means and standard deviations for normally distributed variables whereas for non-normally distributed variables we determined frequencies and proportions. Chi-square or Fisher exact test was applied for categorical variables as appropriate. Data were analyzed using Stata version 12 (StataCorp LLC, College Station, USA). A p-value of $<0.05$ was deemed significant.

\section{Results}

A total of 2914 patients visited CAR-ERU from October 2016 to September 2017. Out of which 30\% were diagnosed with the acute coronary syndrome (ACS) and this included 8\% diagnosed with STEMI, 13\% diagnosed with NSTEMI. Twenty percent were recorded as non-ACS (such as patients with heart failure, valvular disease, arrhythmias and hypertension urgency) cardiac cases (Figure 1).

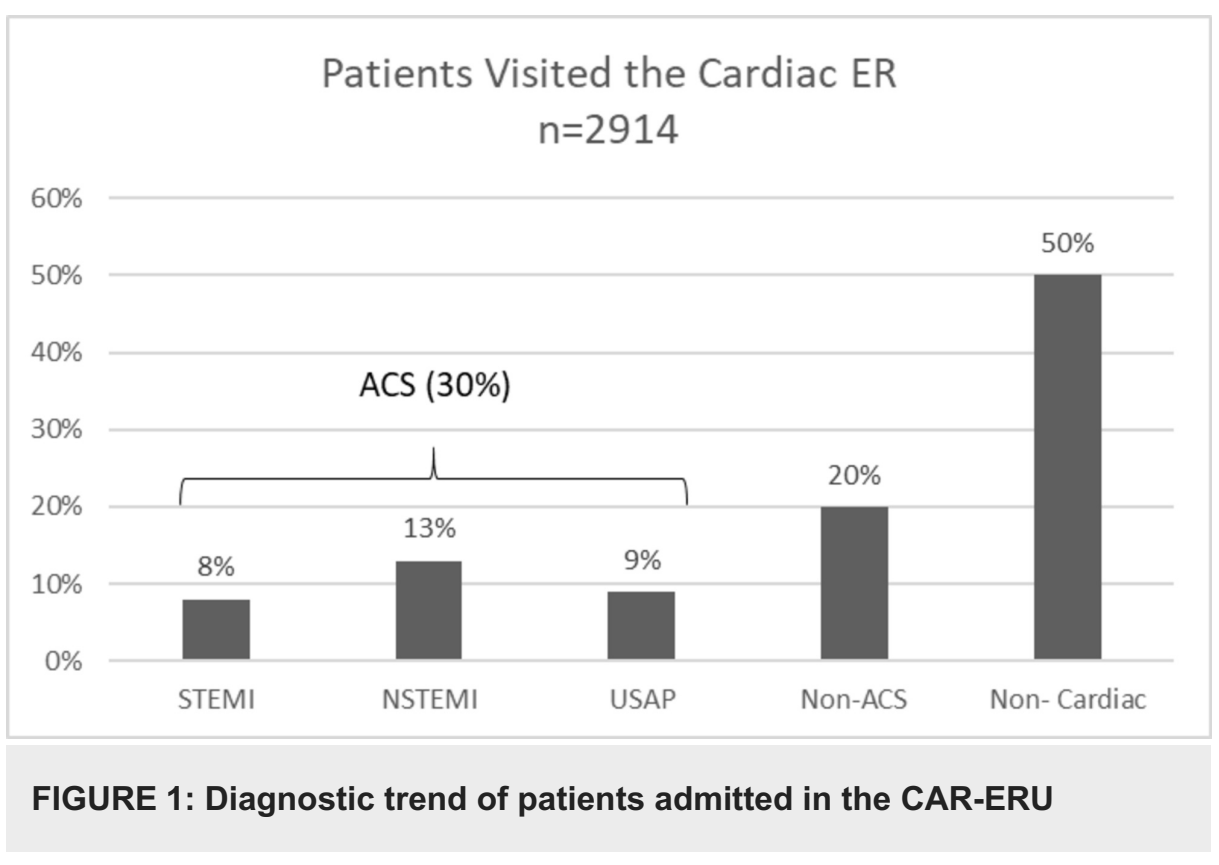




\section{Cureus}

We found that $50 \%(n=1457)$ patients initially suspected of cardiac pathology were finally diagnosed as a non-cardiac case by a specialized and dedicated cardiac team within one hour of their arrival. This resulted in a significant reduction in emergency length of stay of chest pain patients and subsequently the cost paid by the patient. In STEMI, on an average $98.8 \%$ of ECGs were done within 10 minutes of arrival while the first dose of aspirin was given within 60 minutes to an average of $96.5 \%$ of the patients.

The door to balloon time (DBT) for the STEMI patients was less than 90 minutes in $70 \%$ of patients; the international benchmark is $90 \%$ according to the national cardiovascular data registry. The median DBT of CAR-ERU patients was 77 minutes (IQR 60-107 minutes) as compared to our previous years (2010-2014) institutional audit data before the establishment of CAR-ERU showing median DBT of 115 minutes IQR 85 155 with less than $50 \%$ of patients had DBT less than 90 min as shown in Figure 2.

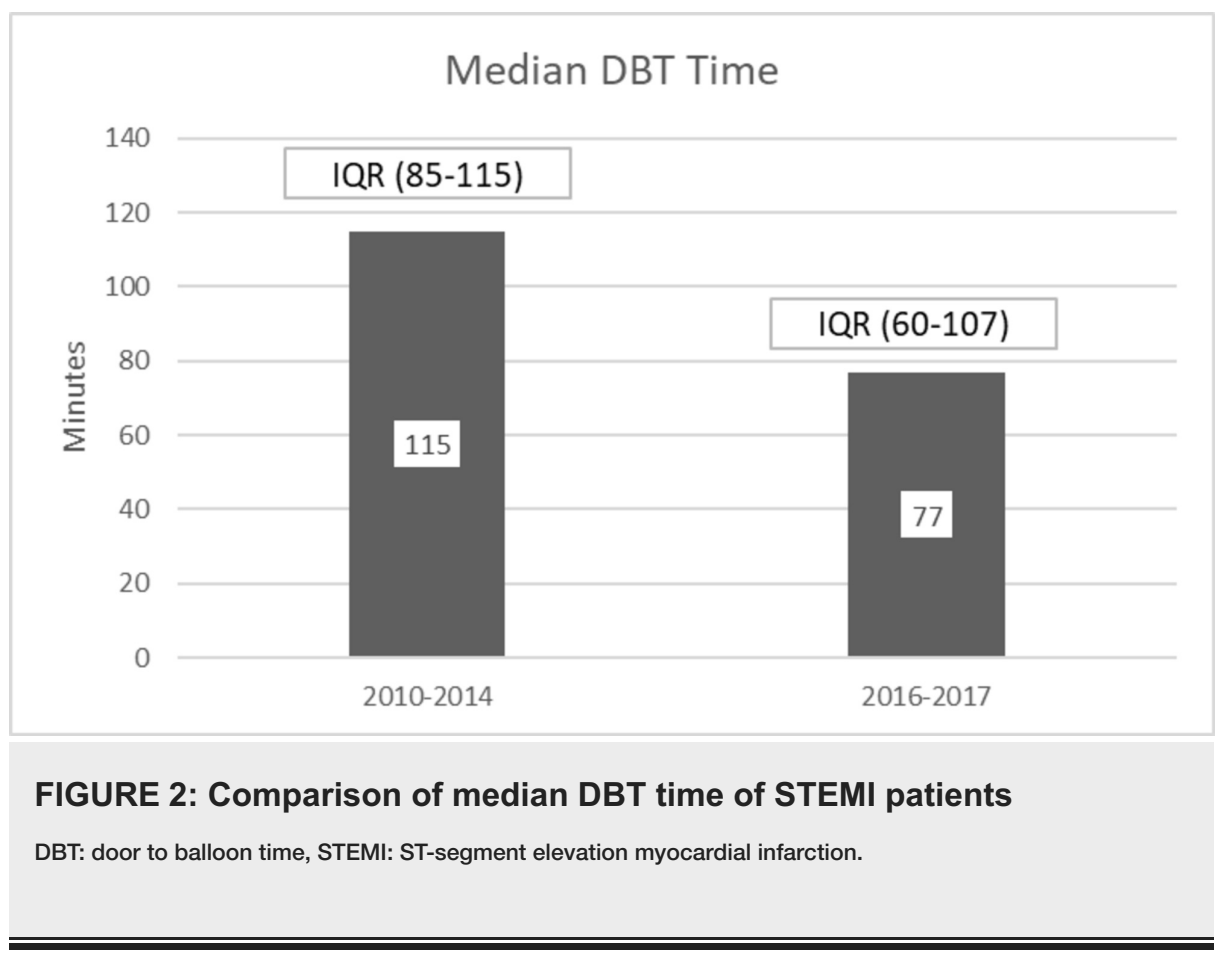

The average ED length of stay of cardiac patients was decreased from 6.1 hours to 2.3 hours (Figure 3) and the cost of care for cardiac patients was reduced to 1040 PKR (\$7) per hour from 2745 PKR (\$18) per hour of ED stay as compared to year 2010-2014 institutional audit data results as shown in (Figure 4).

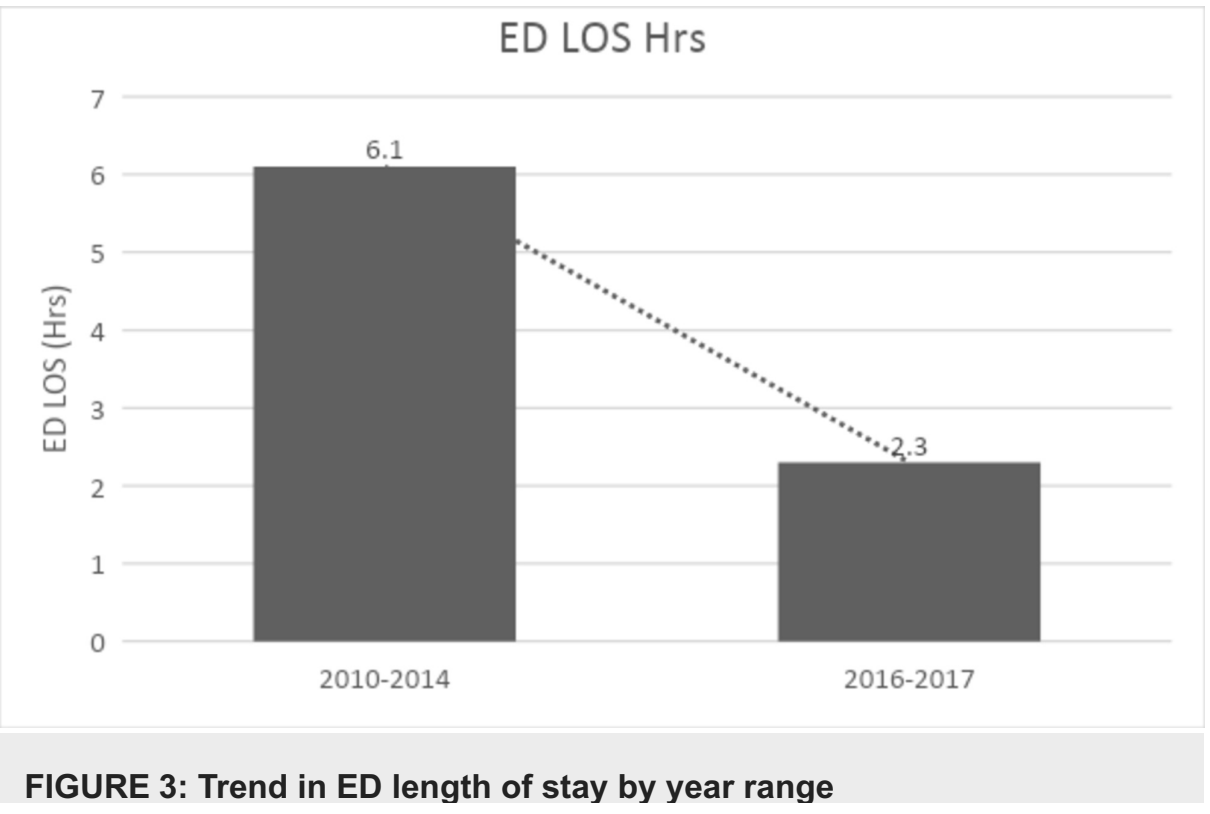




\section{Cureus}

ED: emergency department.

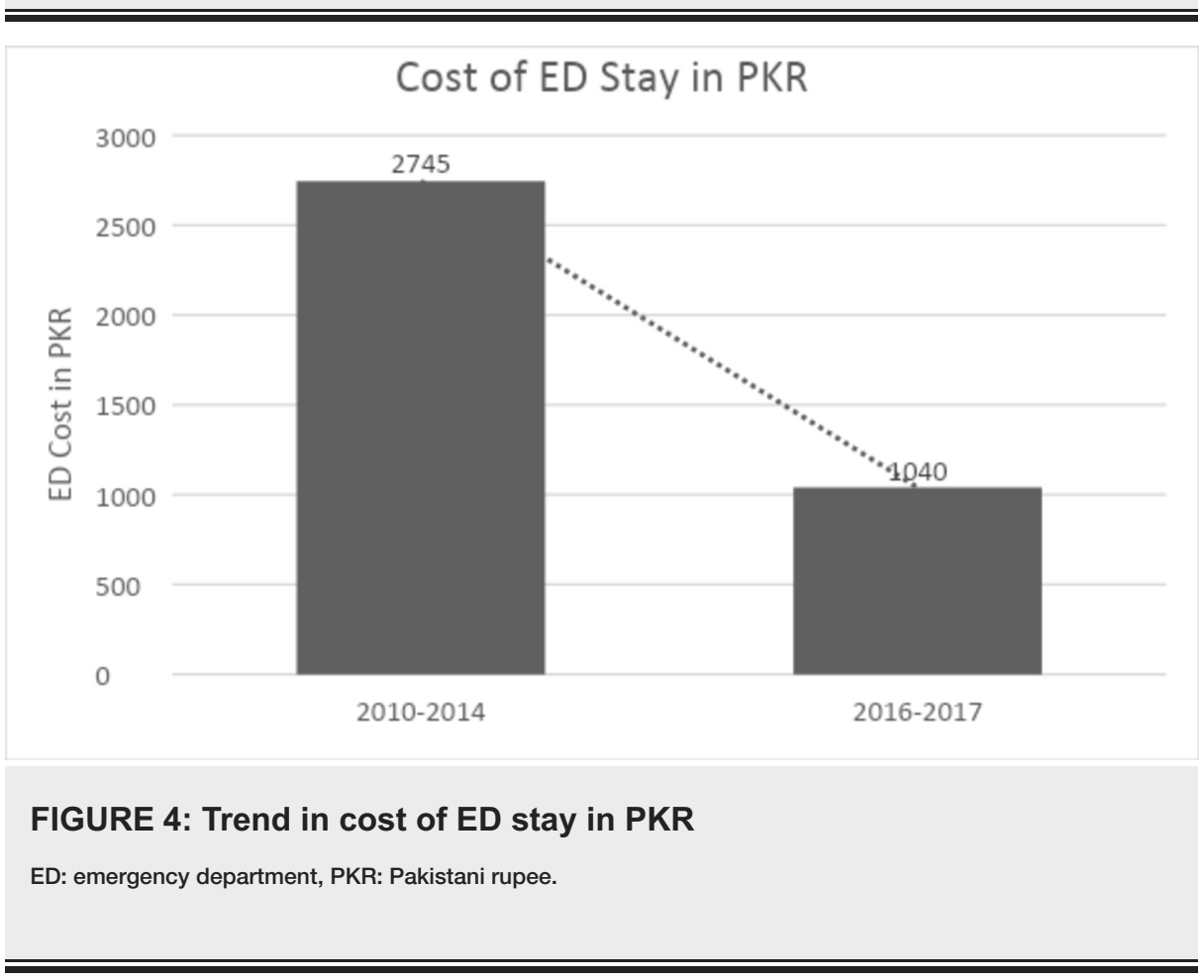

A significant difference in hospital mortality of cardiac patients from $5.4 \%$ to $18 \%$ (p-valve $=0.019$ ) was observed between less than and more than 90 min of DBT, respectively (Figure 5).

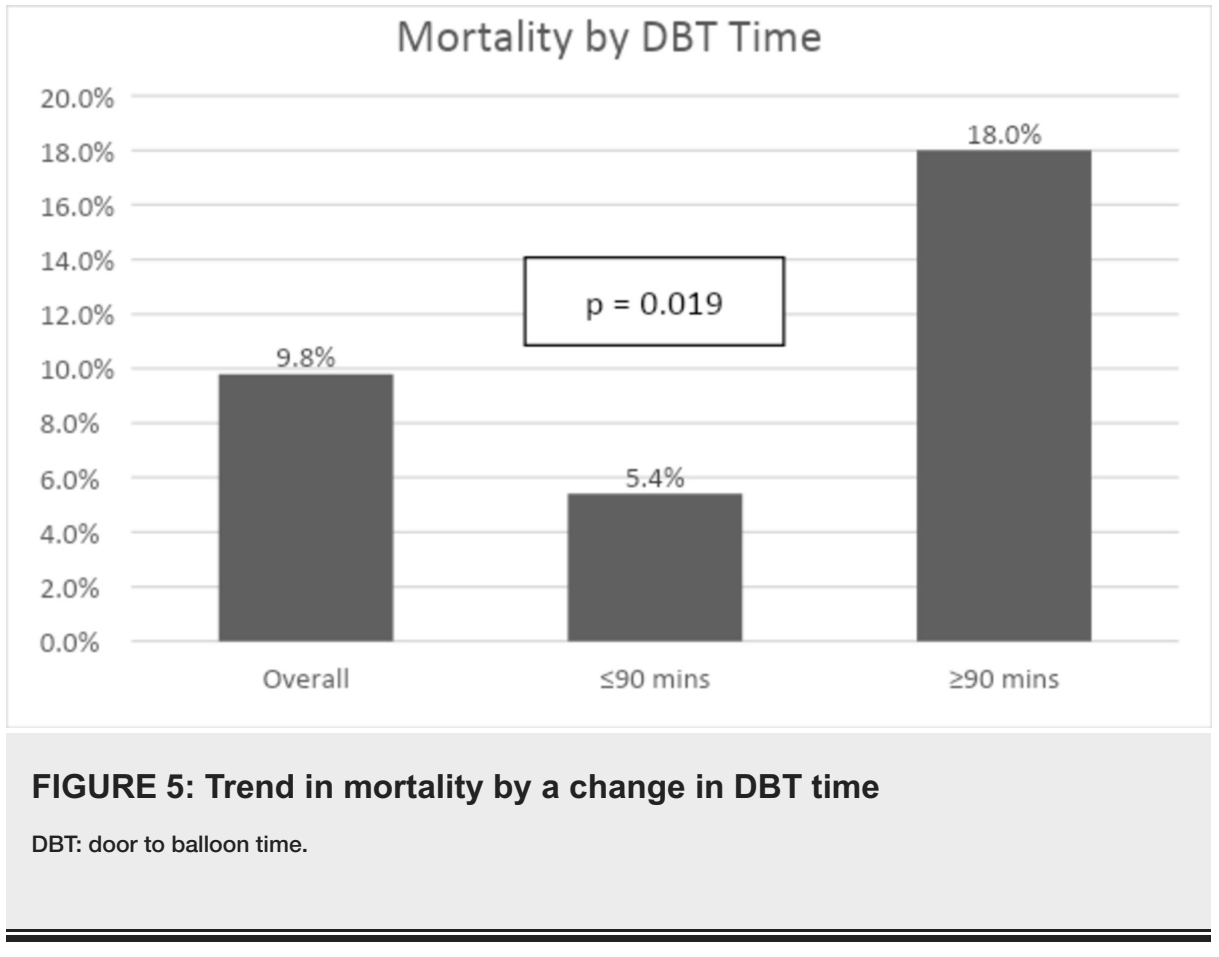

Overall morbidity is defined as a new onset of complications experienced during the hospital stay and includes major and minor morbidity [11] that was $11.6 \%$; however, no significant difference was calculated between the groups ( $\mathrm{p}$-value $=0.142$; Figure 6 ). 


\section{Cureus}

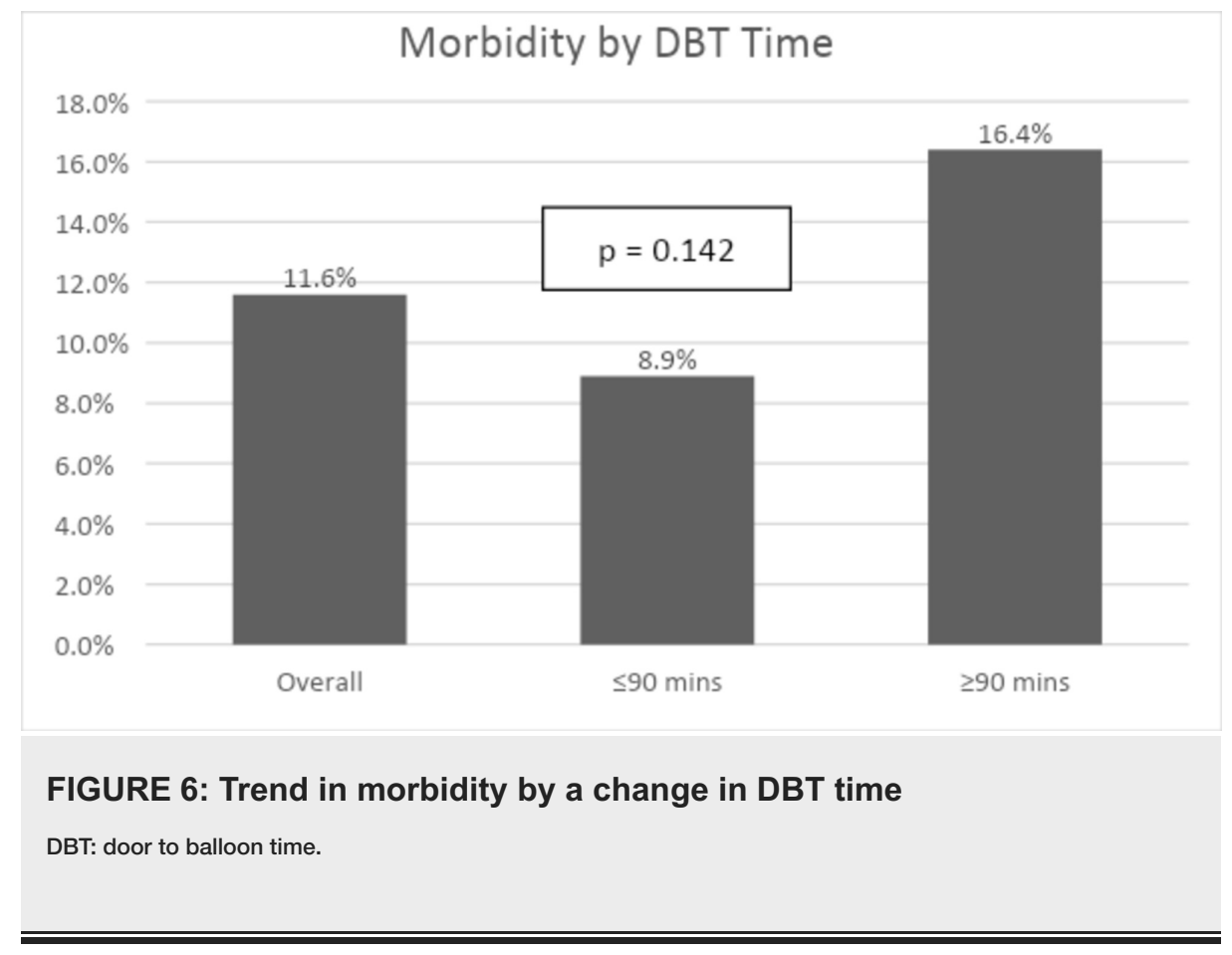

To measure the sustainability of outcomes we continued to collect and analyse major KPIs over the year 2018 and 2019. The major KPIs measured and sustained over the period of 2018-2019 are shown in Figures 7-10.

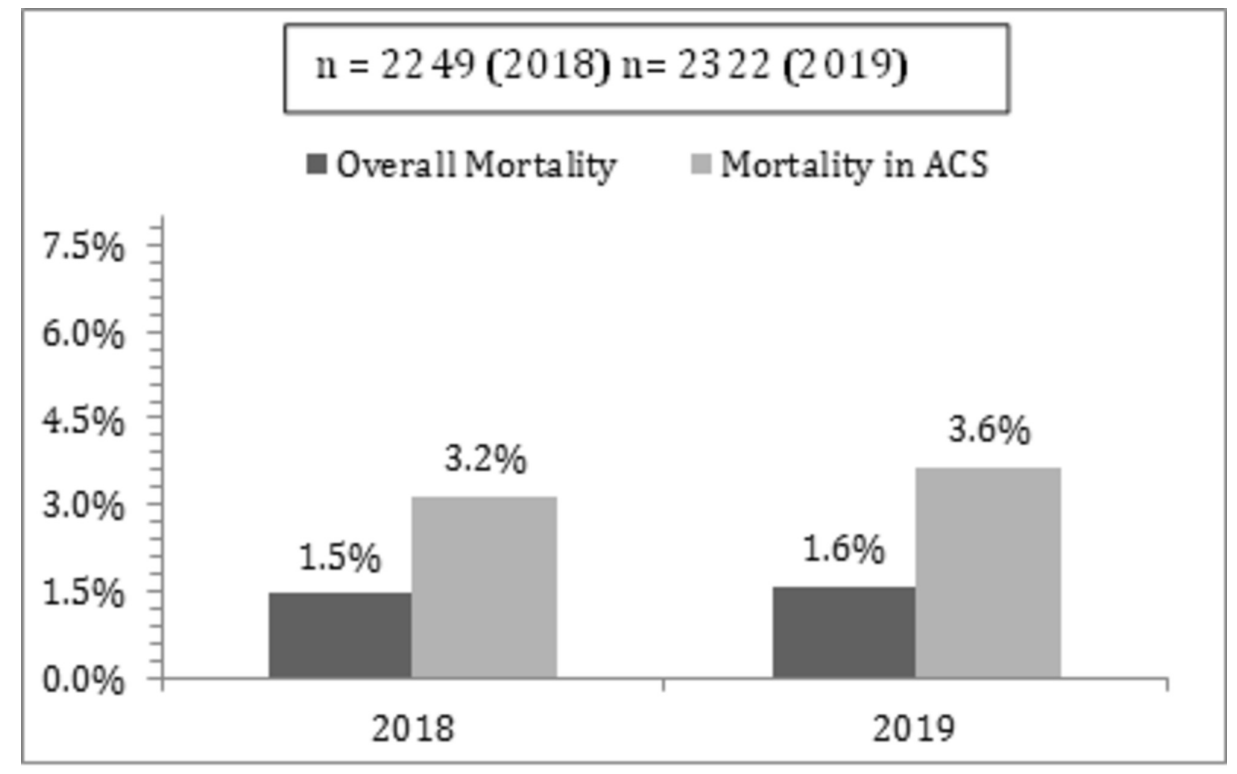

FIGURE 7: Overall and ACS mortality in 2018 and 2019 showing improvement as compared to $5.6 \%$ in 2017

ACS: acute coronary syndrome. 


\section{Cureus}

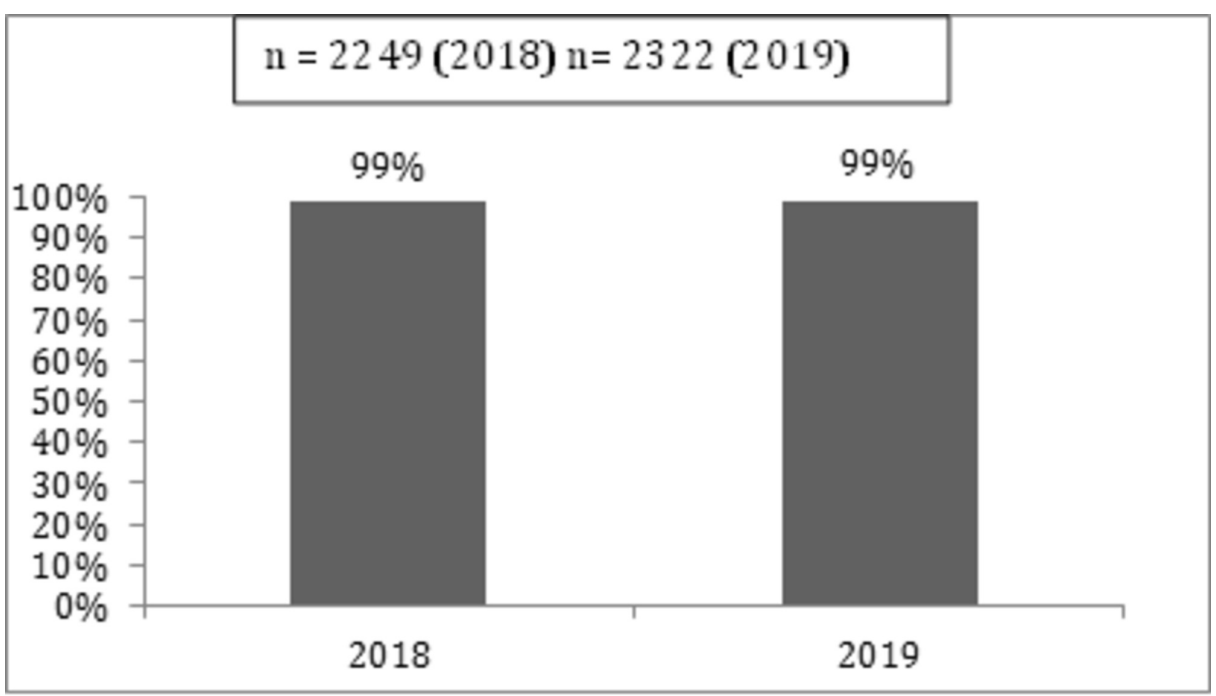

FIGURE 8: ECG within 10 minutes of arrival in CAR-ERU 2018 versus 2019

ECG: electrocardiogram, CAR-ERU: cardiac emergency unit.

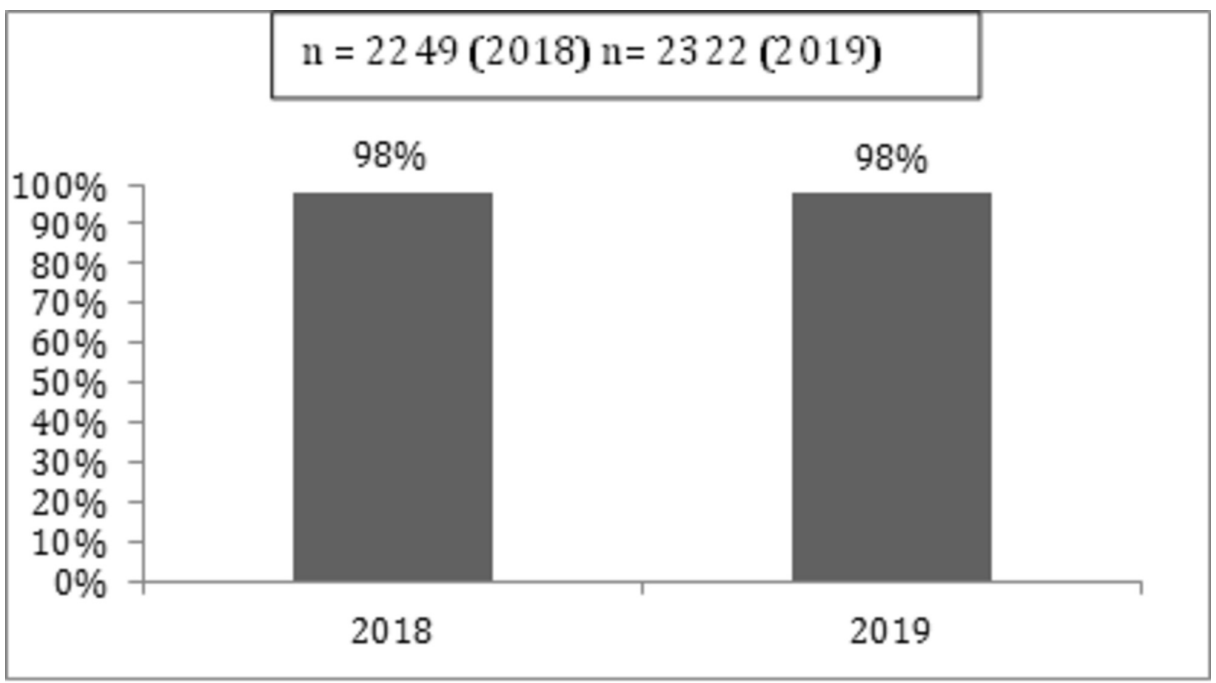

FIGURE 9: Aspirin within 60 minutes of arrival in CAR-ERU 2018 versus 2019

CAR-ERU: cardiac emergency unit. 


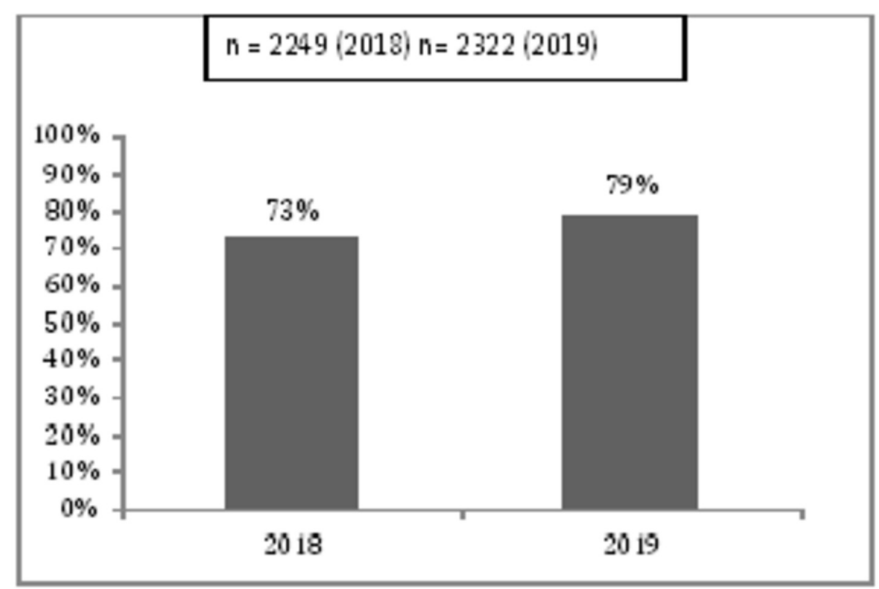

FIGURE 10: Door to balloon time $<90$ minutes 2018 versus 2019

\section{Discussion}

In a recent qualitative study of third world countries like Pakistan, there are many obstacles in the way of providing good quality care to the patients; cost being one of them which not only hinders the delivery of care by healthcare professionals but also the adherence of the patients to their medications [10]. Hence, the quality projects like the establishment of a separate cardiac emergency unit within MED for early identification and management of patients with AMI, not only saves lives but also reduces financial expense and length of stay in ED.

A study done at the Department of Medicine, University of California shows that in spite of providing the latest treatment options, the morbidity and mortality are high in missed myocardial infarction cases, and therefore, they have emphasized a standardized approach to the management of such cases [2]. The substantial decline in mortality cost borne by the patient and length of emergency stay in our data has supported these findings.

The European standard criteria for setting up a separate CAR-ERU as an integral part of MED are already set [11]. The criteria stated that patients with chest pain suspected of AMI should be directly triaged by the cardiac team in CAR-ERU without delay. The structure of our cardiac emergency unit meets with the standard criteria set for the chest pain unit. A dedicated and trained healthcare provider works in CAR-ERU with a strong collaboration among each other. The training for the staff is held every year along with monthly refresher courses.

Pre-hospital care is a neglected area in developing countries; hence, there could be a myriad of impacts on the patient outcome by quality improvement initiatives. A separate cardiac emergency unit can be linked to emergency medical services (EMS), facilitating pre-hospital identification of AMIs which can lead to timely revascularization and myocardial salvage [12].

Although our data have shown promising results, the study has certain limitations. The key performance indicators and outcomes are not stratified according to patient demographic factors, socioeconomic class, type and characteristic of myocardial infarction. Factors responsible for delayed revascularization in AMC, other than the delayed diagnosis in an emergency, such as lack of patient autonomy, family taking a decision on behalf of the patient, literacy rate, gender inequity and many more need to be identified and worked upon. A follow-up for five and ten years would better support the sustainability of parameters.

\section{Conclusions}

The introduction of a dedicated CAR-ERU led to streamline the process of diagnosis and treatment of AMI patients, providing them the best quality of care and decreasing their morbidity, mortality, length of ED stay and financial burden. Not only was this project successful in establishing KPIs but it also showed better results in outcome matrices. The future implications of this quality project seem attainable and, if this is sustained can have an important effect on the patient's quality of life.

\section{Additional Information}

Disclosures 
Human subjects: Consent was obtained by all participants in this study. Ethical Review Comitee issued approval 3163-MED-ERC-14. This is an internal service line data maintained and owned by the quality improvement committee of heart-lung and vascular service line. Animal subjects: All authors have confirmed that this study did not involve animal subjects or tissue. Conflicts of interest: In compliance with the ICMJE uniform disclosure form, all authors declare the following: Payment/services info: All authors have declared that no financial support was received from any organization for the submitted work. Financial relationships: All authors have declared that they have no financial relationships at present or within the previous three years with any organizations that might have an interest in the submitted work. Other relationships: All authors have declared that there are no other relationships or activities that could appear to have influenced the submitted work.

\section{Acknowledgements}

We would like to acknowledge the Department of Emergency medicine The Aga Khan University Hospital Karachi Pakistan for their contribution in the flow process and providing technical support.

\section{References}

1. Hing E, Hall MJ, Xu J: National Hospital Ambulatory Medical Care Survey: 2006 emergency department summary. Natl Health Stat Report. 2008, 1-31.

2. O'Connor RE, Bossaert L, Arntz HR, et al.: Part 9: acute coronary syndromes - 2010 international consensus on cardiopulmonary resuscitation and emergency cardiovascular care science with treatment recommendations. Circulation. 2010, 122:S422-S465. 10.1161/CIRCULATIONAHA.110.985549

3. Leite L, Baptista R, Leitão J, et al.: Chest pain in the emergency department: risk stratification with Manchester triage system and HEART score. BMC Cardiovasc Disord. 2015, 15:48. 10.1186/s12872-0150049-6

4. Razzak JA, Kellermann AL: Emergency medical care in developing countries: is it worthwhile? . Bull World Health Organ. 2002, 80:900-905.

5. Kurji Z, Premani ZS, Mithani Y: Analysis of the health care system of Pakistan: lessons learnt and way forward. J Ayub Med Coll Abbottabad. 2016, 28:601-604.

6. Goldman L, Kirtane AJ: Triage of patients with acute chest pain and possible cardiac ischemia: the elusive search for diagnostic perfection. Ann Intern Med. 2003, 139:987-995. 10.7326/0003-4819-139-12200312160-00008

7. Gale CP, Manda SO, Batin PD, Weston CF, Birkhead JS, Hall AS: Predictors of in-hospital mortality for patients admitted with ST-elevation myocardial infarction: a real-world study using the Myocardial Infarction National Audit Project (MINAP) database. Heart. 2008, 94:1407-1412. 10.1136/hrt.2007.127068

8. Thygesen K, Alpert JS, Jaffe AS, et al.: Third universal definition of myocardial infarction. Circulation. 2012, 126:2020-2035. 10.1161/CIR.0b013e31826e1058

9. Borja Ibanez, Stefan James, Stefan Agewall, et al.: 2017 ESC Guidelines for the management of acute myocardial infarction in patients presenting with ST-segment elevation: the Task Force for the management of acute myocardial infarction in patients presenting with ST-segment elevation of the. Eur Heart J. 2018, 39:119-177. 10.1093/eurheartj/ehx393

10. Legido-Quigley H, Naheed A, de Silva HA, et al.: Patients' experiences on accessing health care services for management of hypertension in rural Bangladesh, Pakistan and Sri Lanka: a qualitative study. PloS One. 2019, 14:e0211100. 10.1371/journal.pone.0211100

11. Masoudi FA, Ponirakis A, de Lemos JA, et al.: Trends in US cardiovascular care: 2016 report from 4 ACC national cardiovascular data registries. J Am Coll Cardiol. 2017, 69:1427-1450. 10.1016/j.jacc.2016.12.005

12. Claeys MJ, Ahrens I, Sinnaeve P, et al.: Editor's Choice - The organization of chest pain units: position statement of the Acute Cardiovascular Care Association. Eur Heart J Acute Cardiovasc Care. 2017, 1:203211. $10.1177 / 2048872617695236$ 